powerful to enable the pictures on the cover to be clearly seen. By throwing the light upon a quickly rotating disk of zinc with a strip of white paper pasted across it, I convinced myself that the phenomenon was a subjective one : the successive images of the white strip always occurred in exactly the same position.

I think these experiments go far towards explaining a matter which must have occurred as a difficulty to many besides myself. Why is it that the illumination produced by a brilliant flash of lightning invariably appears to be of a quivering or intermittent character? We know that the actual duration of a single discharge is sensibly infinitesimal, and both reasoning and laboratory experiments would lead us to believe that a rapid series of discharges between two insulated bodies must at all events be of infrequent occurrence, and due to exceptional circumstances. Yet the quiver of the lightning-flash is proverbial. It would be interesting to ascertain by means of a revolving disk with a single white band across it, whether this is not in most cases a purely subjective phenomenon, due to a succession of after-images.

Wandsworth, June I

\section{Iridescent Crystals of Potassium Chlorate}

With regard to the above crystals, described by Prof. Stokes in NATURE (April 16, p. 565), I should like to suggest, with some diffidence, that the colours may be due, not to a continuous hemitropic crystal-film, but to a series of fine tubular cavities ranged parallel to each other between the two main portions of the crystal, such as not unfrequently occur on a large scale in Iceland spar, and appear to be due to bad fitting (so to speak) of hemitrope-films on the rest of the crystal (see Groth's "Physikalische Krystallographie," p. 44I).

The surfaces of these prismatic cavities, which may be of almost wave-length fineness, would form a series of furrows from whish light would be reflected under the same conditions as from grooved surfaces like those of mother-of-pearl; and, according to the usual laws of interference, we should expect such phenomena to occur as are described by Prof. Stokes: e.g., nonpolarisation of the light, predominance of rays of a particular refrangibility in the reflected beam, and total disappearance of this beam at two azimuths differing by $180^{\circ}$, when the lengthdimension of the tubes lies parallel to the plane of incidence.

I may mention that plates of opal-at any rate those portions which give a uniform colour-afford spectra extremely similar in character to those of the iridescent crystals : definite bright bands in the reflected light, and dark complementary bands in the transmitted light, changing their position in the spectrum with change of incidence. Now the iridescence of opal is pretty well known to be due to the reflection of light from the surfaces of rows of filaments imbedded in the mass (see Sir David Brewster's paper in Brit. Ass. Reports, 1844, part 2, p. 9).

The above hypothesis would also account for Prof. Stokes's observation that the iridescent crystals were best formed when the solution was gently stirred; the molecules then, like a harassed army, being too much disturbed to "fall in" as they should do.

I cannot say that I have yet succeeded in actually bringing out such rows of tubes under the microscope. It is easy to see with a $\frac{1}{5}$-inch power, when the illuminating bean is properly adjusted, a sort of wavy structure at the twin-film (like that seen in opal under the same conditions); but I have not yet made out such definite rows of cavities as would seum to be required to produce the singularly uniform sheet of colour. They may be beyond the power of a microscope altogether.

Eton College

H. G. MADAN

P.S.--Since I wrote the above, Prof. Stokes has very kindly pointed out that opal spectra are fully described by Mr. Crookes in Pros. Roy. Soc., xvii. 448. One opal micro-section which I have gives a spectrum almost exactly like No. 12 in that paper, but the band is slightly less refrangible than the $D$ line at an incidence of $20^{\circ}$.

\section{Pre-Existence and Post-Existence of Thought}

To express any views on these subjects one might well have hesitated some years ago, as hereditary transmission, tolerated as a doctrine, chiefly with regard to the breeding of sheep and dogs, was held to be unphilosophical. Darwin has settled the matter in the domain of science, but perhaps without altogether disposing of prejudices. Mr. Galton and myself have long since dealt with less material aspects of heredity.

What I want to bring on the scene of consideration is the common notion of throwing the great-grandsire. Few now are inclined to doubt about this.

Throwing the grandsire in the case of man may be taken to represent a period of a hundred years of transmission until birth for three generations, and consequently such period of the preexistence of a thought or habit, which is in one's self, and of which there may be an actual register. It is better to select the example of a man, and of a great-grandsire, rather than of a father, because it carries with it a period of remoteness.

Taking then a period of a century for pre-existence in the past, there comes the consideration of vitality of occurrence in the future, as we know of it from the past. Taking a generation for the birth of offspring, and adding to it three generations for a great-grandchild, we have, say, one hundred and fifty years. Adding together the two periods, we obtain an epoch of transmission in pre-existence and of possible existence of two hundred and fifty years.

One such epoch antecedently and another subsequently may be counted in round numbers five hundred years, and we shall see our way to a thousand.

Perhaps after all we know less in detail of the transmission of osseous peculiarities than we assume. There are the means in some cases of examining bony structures of epochs and intervals of $1,000,2,000,5,000$, 10,000 and more years, and we can trace forms of development. What we further want is the power of witnessing the successive individual variations, which is attended with diversity from other beings, and constitutes identity. Though identity may to some extent repose in the process of a bone, in the way in which a vein or sinew has traversed, we have evidence of this rather collectively in a species or a variety, than in the mode in which it periodically or irregularly influences a series of individuals.

The transmiscion of physical peculiarities in the soft tissues is equally permanent, and can be better traced in the individual. There are good examples of it in well-known cases of family features, and of racial types.

We are now familiar with cases of the hereditary transmission of mental qualities, as in that of the Bernouilli family, though perhaps one of the most remarkable instances is that of the Ottoman dynasty, members of which for several hundred years have displayed capacity, and yet here we have only the male elements of transmission, as in the marriages there has been a great mixture of races. It may, however, be that the Turk, the so-called Circassian (sometimes a Daghestani), and the Georgian are more nearly allied than we have been disposed to consider.

The problem now before us may be treated, irrespective of what laws there may be of male or female transmission, as in breeding, the qualities of an ancestor on either side and of either sex may be reproduced, and it is this reproduction and transmission of mental qualities which is to be considered. The mental qualities must be distinguished from the osseous structure or the soft tissues in degree. There is a vast difference in extent, and probably in distinction, between the transmission of some feature and that of conscious or unconscious thought or even of a dream.

My own term of "unconscious thought" I prefer to "unconscious cerebration," because the main distinction between conscions and unconscious thought is chiefly, if not wholly, dependent on the quality of consciousness. One reason for retaining it here is with reference to dreams. Whatever may be the operation of recording other thoughts, dreams are certainly preserved during life as effectually as any kind of thought, although no organ of seeing or hearing is concerned.

If it is remarkable that purely physical properties should be preserved in the germ of a minute animal, it is much more so that any process allied to the operations of thought should be preserved - the influence of events, the influence of dreams, used age after age to constitute the mind of this day. It will be perceived that I am speaking very loosely and vaguely, to some extent of set purpose, to bring under consideration a genera question of long time in the transmission of a mental process, whether connected with what is called instinct or unconscious thought, and without limiting the discussion more than is necessary with postulates or set definitions.

32, St. George's Square, S.W., May 23
Hyde Clarke 